\title{
Spectral Signatures of Canthaxanthin Translocation in the Orange Carotenoid Protein
}

\author{
Natalia B. Pigni, Kevin L. Clark, Warren F. Beck, and José A. Gascón* \\ Cite This: https://dx.doi.org/10.1021/acs.jpcb.0c08756 \\ Read Online
}

ACCESS

Wlll Metrics \& More

Article Recommendations

Supporting Information

ABSTRACT: The orange carotenoid protein (OCP) is involved in the photoprotective processes in cyanobacteria via nonphotochemical quenching. Triggered by blue-green light absorption, the carotenoid chromophore undergoes translocation, displacing around $12 \AA$ from the C-terminal domain (CTD) to the N-terminal domain (NTD). The detailed molecular rearrangements that occur within the carotenoid and the protein during this process remain largely elusive. By using a combination of molecular dynamics, welltempered metadynamics, and hybrid quantum mechanical/molecular mechanical (QM/MM) calculations, we were able to mimic the translocation of the carotenoid from the inactive $\mathrm{OCP}^{\mathrm{O}}$ and obtain metastable red-shifted states in the photoactivation mechanism, replicating the $\lambda_{\max }$ values of reference experimental spectra. In addition, our simulations give insight into the structure of the red-shifted form of the inactive state of OCP.

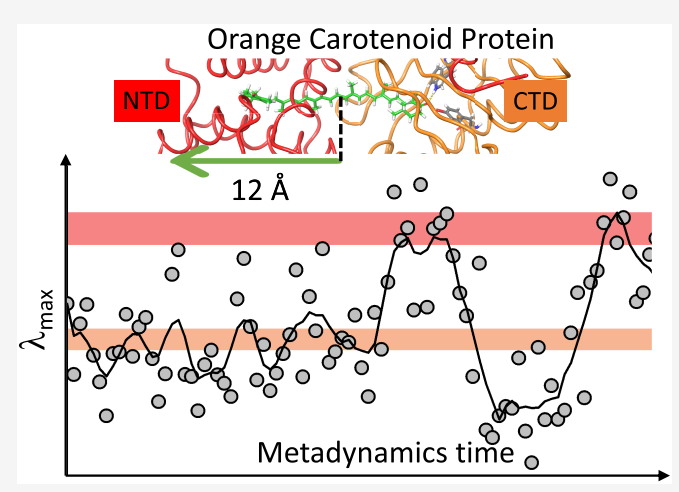

\section{INTRODUCTION}

Photosynthetic organisms have developed efficient ways for light energy capture and storage, accompanied by a refined defense mechanism against varying light conditions. Nonphotochemical quenching (NPQ) protects these organisms from the potentially damaging effects of excess light. In cyanobacteria, NPQ is facilitated by the orange carotenoid protein (OCP). ${ }^{1}$ OCP is a $35 \mathrm{kDa}$ water-soluble protein consisting of an all $\alpha$-helical $\mathrm{N}$-terminal domain (NTD) and a mixed $\alpha$-helical $/ \beta$-sheet C-terminal domain (CTD) (Figure 1A). Encapsulated between these two domains, a ketocarotenoid interacts with the protein environment through hydrogen bonds and hydrophobic contacts. ${ }^{2,3}$ Under normal light conditions, OCP is found in its inactive form $\left(\mathrm{OCP}^{\mathrm{O}}\right)$ characterized by an orange coloration in solution. After photoactivation by blue-green light, OCP undergoes a series of reversible structural modifications leading to the active form known as $\mathrm{OCP}^{\mathrm{R}}$ with a typical red coloration. $\mathrm{OCP}^{\mathrm{R}}$ exerts its function through the interaction with the phycobilisome (PBS), being responsible for quenching the excess of energy. ${ }^{4}$ Either spontaneously or facilitated by the fluorescent recovery protein (FRP), $\mathrm{OCP}^{\mathrm{R}}$ is then reconverted to $\mathrm{OCP}^{\mathrm{O}} .{ }^{5} \mathrm{OCP}$ can bind different carotenoids, including $3^{\prime}$-hydroxyechinenone $\left(3^{\prime}-\mathrm{hECN}\right)$, echinenone, and canthaxanthin (CAN, Figure 1B). All of these ketocarotenoids have a common carbonyl group that stabilizes the $\beta 1 \beta$-ionone ring of $\mathrm{OCP}^{\mathrm{O}}$ through hydrogen bonds with two key residues from the CTD, Tyr201 and Trp288. ${ }^{6}$ Stabilization and disruption of this pair of hydrogen bonds is apparently essential for the photoactivation cycle because these hydrogen-bonding interactions are highly conserved in OCP structures from different cyanobacteria. ${ }^{3}$
Recent studies of the fluorescence spectra from $3^{\prime}-\mathrm{hECN}$ and $\mathrm{CAN}$ in $\mathrm{OCP}^{\mathrm{O}}$ show that the two ketocarotenoids differ in their ability to form an additional hydrogen bond between the $\beta 2 \beta$-ionone ring with Leu37 in the NTD. Interestingly, the referenced studies led to the conclusion that large-amplitude motions of the $\beta 2$-ring contribute to the earliest events following absorption of light that leads to photoactivation to the $\mathrm{OCP}^{\mathrm{R}}$ state.

Protein structural details of the photoactivation mechanism have been recently described using time-resolved electronic and vibrational spectroscopy. ${ }^{8}$ Optical excitation of the midvisible absorption band populates the $S_{2}$ state of the carotenoid followed by ultrafast internal conversion to the dark $S_{1}$ state, which is coupled to an intramolecular charge-transfer (ICT) state. The $S_{1} /$ ICT state further decays to the ground state. With a yield of $\sim 1.5 \%$, this overall process results in the photoproduct P1 (Figure 1C), which is characterized by the rupture of hydrogen bonds between the carotenoid and Tyr201 and Trp288. Around 40\% of this intermediate photoproduct is temporarily stabilized by protein rearrangements in the NTD side (photoproduct P2). These movements lead further on $(\sim 10 \mu \mathrm{s})$ to a remarkable $12 \AA$ translocation of the carotenoid toward the NTD. ${ }^{9}$ The resulting photoproduct

Received: September 25, 2020

Revised: November 25, 2020 
A

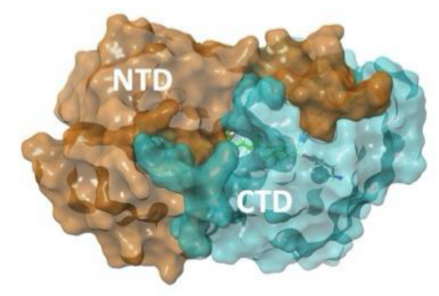

B<smiles>CC1=C(C)[C@H](/C=C/C(C)=C/C=C/C(C)=C/C=C/C=C(C)/C=C/C=C(C)/C=C/C2=C(C)C(=O)CCC2(C)C)C(C)(C)CCC1=O</smiles>

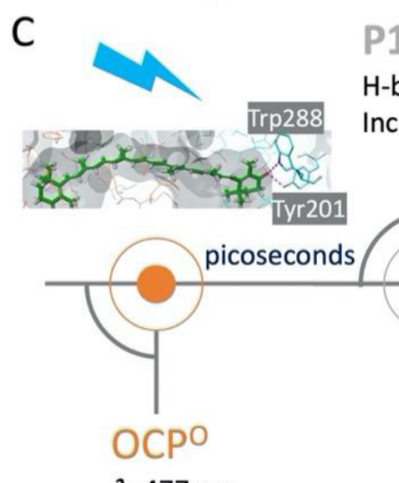

$\lambda=477 \mathrm{~nm}$
P1 H-bonds broken Increased planarity

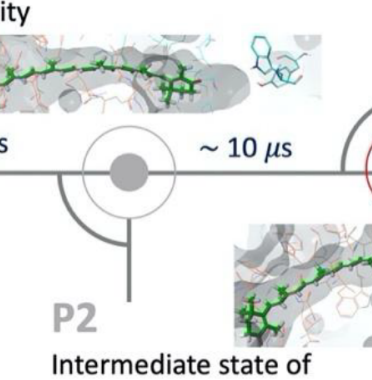
Intermediate state of CAN translocation

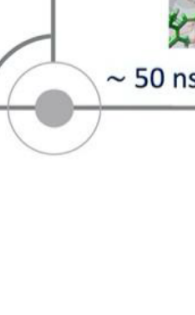

P3

Full $12 \AA$

translocation

CAN translocation

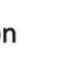

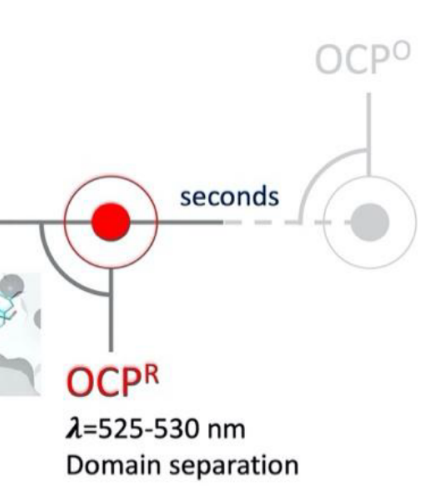

Figure 1. (A) Structure of OCP and its two domains. (B) Structure of canthaxanthin (CAN). The $\beta$-ionone ring bound by the NTD is labeled $\beta 2$, whereas that bound by the CTD is labeled $\beta 1$. (C) Timeline of OCP photoactivation based on the intermediate states proposed by Konold et al. ${ }^{8}$ P1 structure is unknown; models shown for P2 and P3 have been obtained from our simulations, vide infra.

(P3) shows a UV-vis spectrum with a close resemblance to that reported for $\mathrm{OCP}^{\mathrm{R}}$. Lastly, on a time scale longer than 1 $\mathrm{ms}$, the two domains separate, leading to the fully active $\mathrm{OCP}^{\mathrm{R}}$ state. $^{10-13}$

The structure of $\mathrm{OCP}^{\mathrm{O}}$ bound to canthaxanthin in solution is characterized by an absorption spectrum with a $\lambda_{\max }$ at $\sim 477$ nm. ${ }^{14,15}$ After photoactivation, OCP ${ }^{\mathrm{R}}$-CAN shows a red-shifted $\lambda_{\max }$ at $\sim 525-530 \mathrm{~nm} .{ }^{9,15}$ The intermediate states (P1-P3) are also reported to have overall red-shifted spectra with respect to the $\mathrm{OCP}^{\mathrm{O}}$ steady-state. ${ }^{8}$ There are several crystal structures of $\mathrm{OCP}^{\mathrm{O}}$ available, such as $\mathrm{OCP}^{\mathrm{O}}$ bound to $\mathrm{CAN}$ (PDB: 4XB5), but no structural information is available for any of the intermediate photoproducts or the fully active $\mathrm{OCP}^{\mathrm{R}}$. However, the structure of the isolated NTD bound to CAN, known as red carotenoid protein (RCP), was successfully crystallized and described in 2015 (PDB: 4XB4) by Kerfeld's group, representing a valuable target model of $\mathrm{OCP}^{\mathrm{R}}$. In fact, RCP in solution displays a UV-vis spectrum almost identical to that of $\mathrm{OCP}^{\mathrm{R}}$ with a $\lambda_{\max }$ reported at $\sim 525-530 \mathrm{~nm} .^{9,14}$

Despite the progress in describing many aspects of the photoactivation mechanism, there are still a number of open questions about the various stages of the cycle, especially regarding the detailed carotenoid rearrangement that disrupts the hydrogen-bond network, the structural dynamics of carotenoid translocation, and domain separation. 8,16

In this work, we elucidate the structural changes that might occur within the protein as the carotenoid emerges into the NTD domain. While the detailed structural changes that occur during the formation of $\mathrm{P} 1$ still remain elusive to experimental and theoretical characterization, we construct a path that directly connects the inactive $\mathrm{OCP}^{\mathrm{O}}$ structure with a P2-like state, followed by a P3-like state, where the carotenoid is displaced by $12 \AA$ toward the NTD side. This pathway represents the hypothetical process where the carotenoid translocates from its inactive state $\mathrm{OCP}^{\mathrm{O}}$ to the red state $\mathrm{OCP}^{\mathrm{R}}$ in the dark. To accomplish this, we perform well- tempered metadynamics simulation to steer the carotenoid from CTD to NTD. Metadynamics has been successfully applied to tackle questions in different fields including materials science, chemical reactions, and biomolecular dynamics. ${ }^{17}$ As an illustrative example, its application has provided insights to understand ligand translocation mechanisms in protein gorges/channels. ${ }^{18}$ With regard to the study of OCP, the use of this methodology has been recently reported to enhance the sampling of CAN conformational freedom in RCP, with an exclusive focus on the geometry of rings $\beta 1$ and $\beta 2 .^{16}$

The metadynamics simulations are combined with QM/ MM calculations to characterize the spectral signatures of the different states of the translocation process. We further offer an explanation for the experimentally observed heterogeneity of the inactive state of $\mathrm{OCP}^{\mathrm{O}}$, which presents a red-shifted spectral component.

\section{COMPUTATIONAL METHODS}

System Preparation. The crystal structures of $\mathrm{OCP}^{\mathrm{O}}$ (PDB: 4XB5) and RCP (PDB: 4XB4) ${ }^{9}$ containing the protein and the carotenoid were processed with Maestro (Schrödinger 2019-4). Water molecules and glycerol were deleted. For the dimeric structure of RCP, only one monomer was kept (chain A). The Protein Preparation application was used to preprocess the complex, assigning bond orders, adding hydrogens, and filling in missing loops with Prime (residues 51-59 in RCP). For the alternative positions of side chains reported in the crystal structures, the most populated ones were kept. The particular residues for each structure, with the respective occupancy value of the selected position given between parentheses, were the following: Glu118 (0.59) for OCP; Cys84 (0.79), Arg112 (0.53), and Ser147 (0.51) for RCP. Optimization of hydrogen-bond assignment was performed with default parameters $(\mathrm{pH} \mathrm{7.0)}$, and a restrained 
minimization was applied for hydrogens only. A $10 \AA$ buffer size, orthorhombic box was constructed using the TIP3P water model, ${ }^{19}$ and volume minimization was applied with a resulting number of water molecules equal to 5814 and 10121 for RCP and OCP, respectively. A number of $\mathrm{Na}^{+}$ions were added to neutralize the negative charge of the system. All preliminary minimizations and MD simulations used the force field OPLS3e. ${ }^{20}$

Molecular Dynamics and Metadynamics. MD simulations were run for solvated systems on Nvidia GPU hardware with Desmond (Schrödinger 2019-4). Prior to MD production, the default relaxation protocol was performed (detailed in SI). Classical MD simulations were run for a total time of 1000 ns using the NPT ensemble ( $300 \mathrm{~K}, 1 \mathrm{~atm})$. Snapshots were extracted every $20 \mathrm{~ns}$ and fed into QSite for QM/MM calculations. Additional information about the MD analysis, including root mean square deviation (RMSD) calculation, description of protein-ligand interactions, ligand solvent accessible surface area (SASA), and torsions data, is reported in SI.

WT-metadynamics simulations were run using the solvated system prepared for $\mathrm{OCP}^{\mathrm{O}}$. The collective variable $(\mathrm{CV})$, to drive the displacement of CAN, was defined as the distance from the center of mass of 5 carbon atoms belonging to 5 residues on the NTD side (Glu34-C $\gamma$, Thr80-C $\beta$, Cys84-C $\beta$, Pro126-C $\alpha, \operatorname{Tyr} 129-\mathrm{C} \delta 1$ ) to $\mathrm{C}^{\prime}$ on the $\beta 2$-ring of CAN (Figure 1B). A wall of $20 \AA$ was set as a limit for the explored coordinate space. This $\mathrm{CV}$ serves as an effective reaction coordinate for the translocation process and the protein response. The width of the Gaussian potential was $\sigma=0.06$, and the initial height $W_{0}=0.03 \mathrm{kcal} \mathrm{mol}^{-1}$. The deposition time was $\Delta t=0.2 \mathrm{ps}$, and the bias factor of WT-metadynamics was set to $\gamma=10$. There were 20 repetitions run varying the seed randomly. The total time for each metadynamics simulation was 100 ns. The ensemble class and relaxation protocol were the same as those applied for classical MD. Snapshots were taken every $1 \mathrm{~ns}$ to perform the electronic structure calculations and to analyze the carotenoid geometry and protein-ligand interactions.

Electronic Structure Calculations. Quantum mechan$\mathrm{ical} /$ molecular mechanical (QM/MM) calculations were run on snapshots extracted from $\mathrm{MD}$ and metadynamics simulations. Solvent and ions were removed. The QM region was defined as CAN while the rest of the protein was at the MM-OPLS3e level. Since the force field largely overestimates the bond length alternation (BLA) of the carotenoid, a local geometry optimization of CAN was performed leaving the rest of the protein frozen. This optimization was performed using QSite (Schrödinger 2019-3) at the DFT B3LYP/LACVP* level. Excited state energies were then calculated on the optimized structures with the Tamm-Dancoff approximation at the CAM-B3LYP/LACVP* level. No ad hoc corrections, shifts, or parametrizations of any kind were employed to fit the calculated TDDFT values with experimental data. An important reason for choosing CAM-B3LYP as the functional is that it is one of the best at reproducing the correct excitation energy of the isolated CAN in solvent, which ranges between 470 and $490 \mathrm{~nm}^{21,22}$ Indeed, we made a comparison with a number of other functionals including SVWN5, BP86, VSXC, BHHLYP, LC-WHPBE, X3LPY, and B3LYP, and we found that CAM-B3LYP was the best at reproducing the solution phase results. This benchmark was done using an optimized geometry at the B3LYP geometry and a conductor-like screening model with methanol as the implicit solvent.

Protein solvation effects were treated using the PoissonBoltzmann implicit solvent method implemented in QSite. This combination of two different levels of DFT and/or basis sets, one for ground state optimization and the other for excited state energies, has been successfully used before, particularly in the context of carotenoids. ${ }^{23-25}$ The incorporation of solvent (via Poisson-Boltzmann in this case) is essential to reproduce the experimental values of UV-vis absorption. Such a substantial solvent effect has been pointed out recently by Guberman-Pfeffer et al. in the multichromophoric peridinin-chlorophyll a-protein (PCP). ${ }^{26}$ As shown in the SI, use of the explicit waters from MD produces very similar results (Table S2). The average excitation for both $\mathrm{OCP}^{\mathrm{O}}$ and $\mathrm{RCP}$ differs by approximately $4 \mathrm{~nm}$, with the explicit case being slightly more blue-shifted in both OCP and RCP.

One-sample $t$ tests were run to analyze the results from the optimized MD snapshots of the reference models, RCP and $\mathrm{OCP}^{\mathrm{O}}$, calculating the confidence intervals at a level of $95 \%(\mathrm{~N}$ $=51$, for each MD). Smoothing of metadynamics data was done by using a moving average of 5 points. The method used to calculate bond length alternation (BLA) is described in the Supporting Information.

\section{RESULTS AND DISCUSSION}

Validation of Reference Models: $\mathrm{OCP}^{\circ}$ and RCP. The crystal structures of $\mathrm{OCP}^{\mathrm{O}}-\mathrm{CAN}$ (PDB: 4XB5) and RCP-CAN (PDB: 4XB4) were used as the starting points to run our $\mathrm{MD}$ simulations and QM/MM calculations. UV-vis experimental data previously reported in the literature were taken as the reference values to validate our methodology. Since the protein system exists in a dynamical equilibrium between multiple conformations, we aimed to obtain a finite-temperature thermodynamics average of the strongest $S_{0} \rightarrow S_{2}$ absorption. In order to obtain a sampling of representative conformations of both complexes, we performed 1000 ns long classical MD simulations for the prepared systems of $\mathrm{OCP}^{\mathrm{O}}$ and RCP. Previous studies have applied especially developed force fields to describe carotenoid dynamics, allowing for a direct calculation of QM-derived electronic properties from $\mathrm{MD}$ snapshots. $^{27-29}$ In our approach, we maintained the general parametrization of the OPLS3e force field, but we locally minimized only the carotenoid for each snapshot under a QM/ MM treatment. Notwithstanding, the most recent version of OPLSA (OPLS3e) incorporates an extended torsional parametrization as well as optimized partial charge assignment. ${ }^{20}$ Taking into account only the polyene chain, the in-place RMSD before and after QM/MM minimization is, on average, $0.28 \AA$. Thus, the local QM/MM minimization produces very small displacements within the protein cavity.

The MD of $\mathrm{OCP}^{\mathrm{O}}$ shows a stable RMSD for the protein $(\sim 1.5 \AA)$ and the ligand $(\sim 1 \AA)$ during the entire simulation (Figure $S 1$ ). The most frequent protein-ligand interactions are the hydrogen bonds with Tyr201 and Trp288, as well as hydrophobic contacts located mainly on the NTD side (Tyr44, Trp110) (Figure S3). On the other hand, in RCP, the RSMD stabilizes around $2.5 \AA$ for both the ligand and the protein (Figure S2). In this case, the carotenoid interacts mostly through hydrophobic contacts between residues of the NTD cavity and the isoprenoid chain, while both rings are exposed to solvent (Figure S4), which explains the enhanced motional 
A

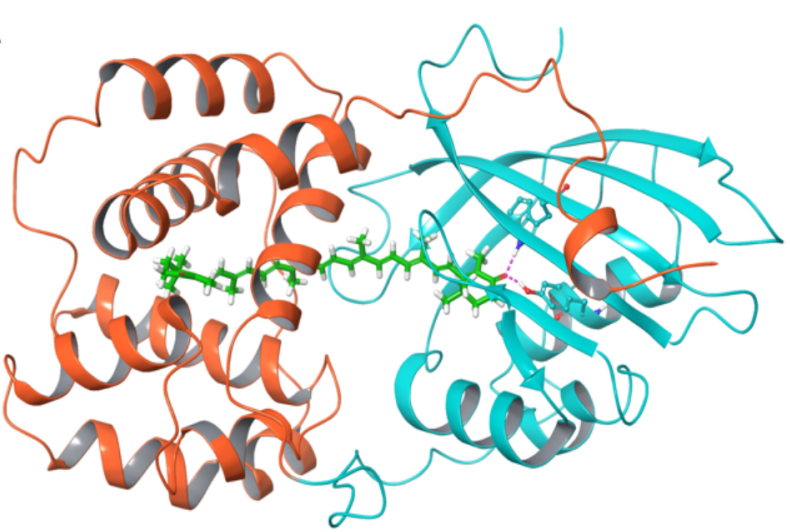

B

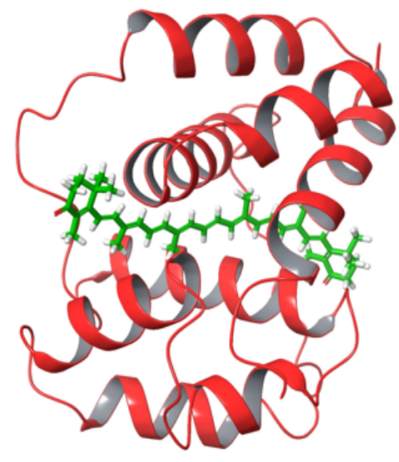

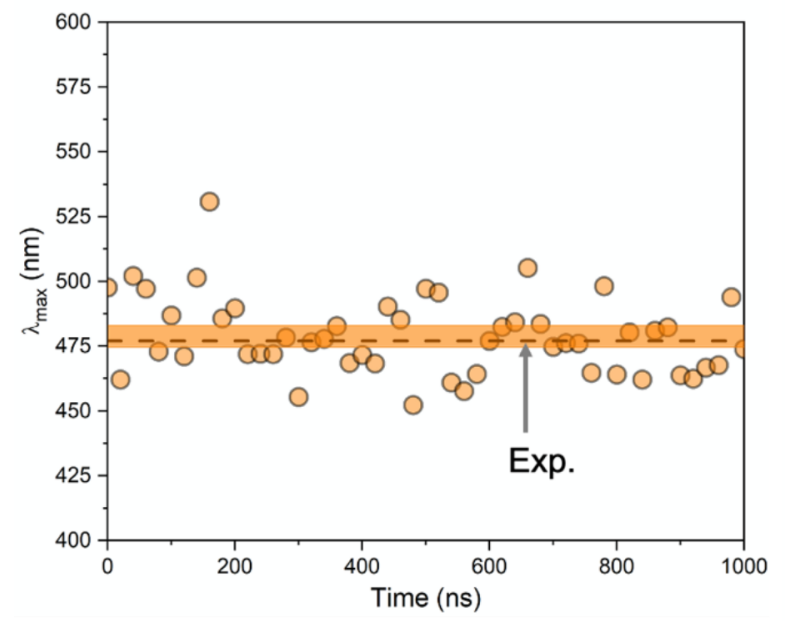

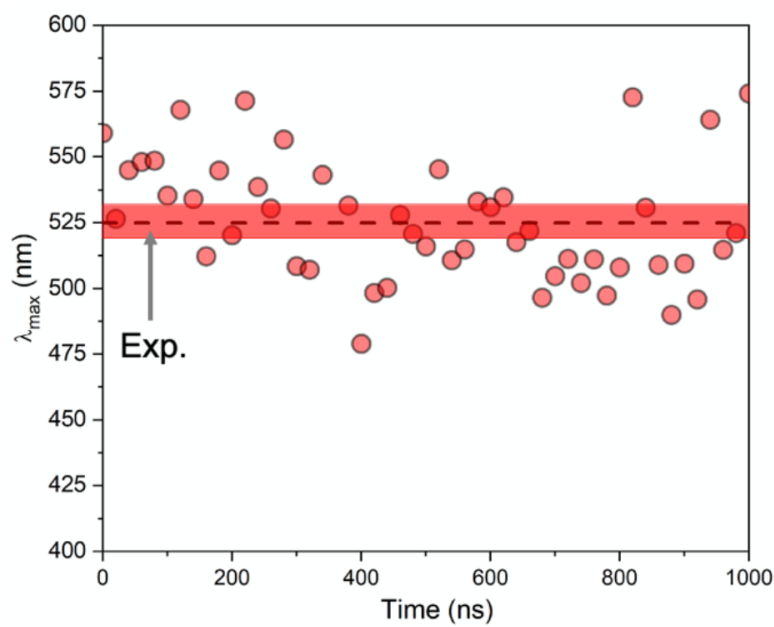

Figure 2. Structures of $\mathrm{OCP}^{\mathrm{O}}-\mathrm{CAN}(\mathrm{A})$ and RCP-CAN (B). The lower panels show the results of the calculated $\lambda_{\max }$ for each locally optimized snapshot extracted from the MD. Confidence intervals (95\%) for both models are shown with orange and red shaded areas, respectively. Dashed lines indicate the experimental $\lambda_{\max }$ reported in the literature for both structures.

\section{Table 1. QM Calculations on Optimized Structures from MD for $\mathrm{OCP}^{\mathrm{O}}$ and RCP Models ${ }^{a}$}

\begin{tabular}{ccccccc} 
model & $\lambda_{\max }(\mathrm{nm})$ & \multicolumn{2}{c}{ confidence interval $(95 \%)$} & SD & BLA & exptl $\lambda_{\max }(\mathrm{nm})^{14}$ \\
OCP $^{\mathrm{O}}$-CAN & 478.7 & 474.5 & 483.0 & 15.1 & $0.073 \pm 0.004$ & 477.0 \\
RCP-CAN & 525.5 & 519.0 & 532.0 & 23.0 & $0.056 \pm 0.007$ & 525.0
\end{tabular}

${ }^{a}$ Results are expressed as the mean of 51 snapshots. A one-sample $t$ test was used to obtain the confidence intervals. Bond length alternation (BLA) is expressed as an average \pm standard deviation.

freedom of the protein and ligand. As expected from the visual inspection of both structures, the ligand SASA is significantly higher in RCP than in $\mathrm{OCP}^{\mathrm{O}}$ (Figure S5). From the resulting MD trajectories, we exported 51 snapshots evenly distributed along the simulation time (every $20 \mathrm{~ns}$ ) for each model. After the deletion of solvent and ions, the structures were optimized using QSite, with the carotenoid included in the QM part of the system, and with the protein frozen. Subsequently, single points were run on each optimized snapshot to obtain excitation energies. Remarkably, despite the variability observed, the confidence intervals are within $2 \%$ of the computed average and reproduce the experimental values reported in the literature (Figure 2, Table 1). These results help validate our computational methodology.

The degree of conjugation of a carotenoid's isoprenoid chain is known to have a strong influence on its electronic properties and excitation energies. ${ }^{27}$ Bond length alternation (BLA) is a parameter that describes such conjugation. In addition, the torsions connecting the isoprenoid chain with the end-rings indirectly affect conjugation. ${ }^{7,14,29,30}$ Our results confirm the direct correlation between the BLA and the calculated $\lambda_{\max }$ for both models, showing a linear correlation with $R^{2}=0.85$ for $\mathrm{OCP}^{\mathrm{O}}$, and $R^{2}=0.95$ for RCP (Figure S6). Dihedrals on both end-rings (Figure 3C) have a higher conformational freedom in RCP, where both ends of the carotenoid are exposed to solvent, while in $\mathrm{OCP}^{\mathrm{O}}$ the protein environment restricts those torsions affecting the electronic properties of the system and lowering the degree of conjugation. ${ }^{16}$ In our MD trajectory, RCP is mostly trapped in one of the free-energy minima showing average values of $\beta 1$-dihedral $=45^{\circ} \pm 7^{\circ}$ and $\beta 2$ dihedral $=159^{\circ} \pm 27^{\circ}$ for the optimized snapshots. This configuration corresponds to a nominal $s$-cis for $\beta 1$ and $s$-trans for $\beta 2$ (as observed in the crystal structure). The large deviations observed for the $\beta 2$-dihedral are caused by a small fraction of states $(\sim 6 \%)$ exploring the $s$-cis and $\sim 90^{\circ}$ configurations. In particular, it has been previously demonstrated that the combination of an $s$-cis $\beta 1$-dihedral with an $s$ trans $\beta 2$-dihedral represents only one of several possible 

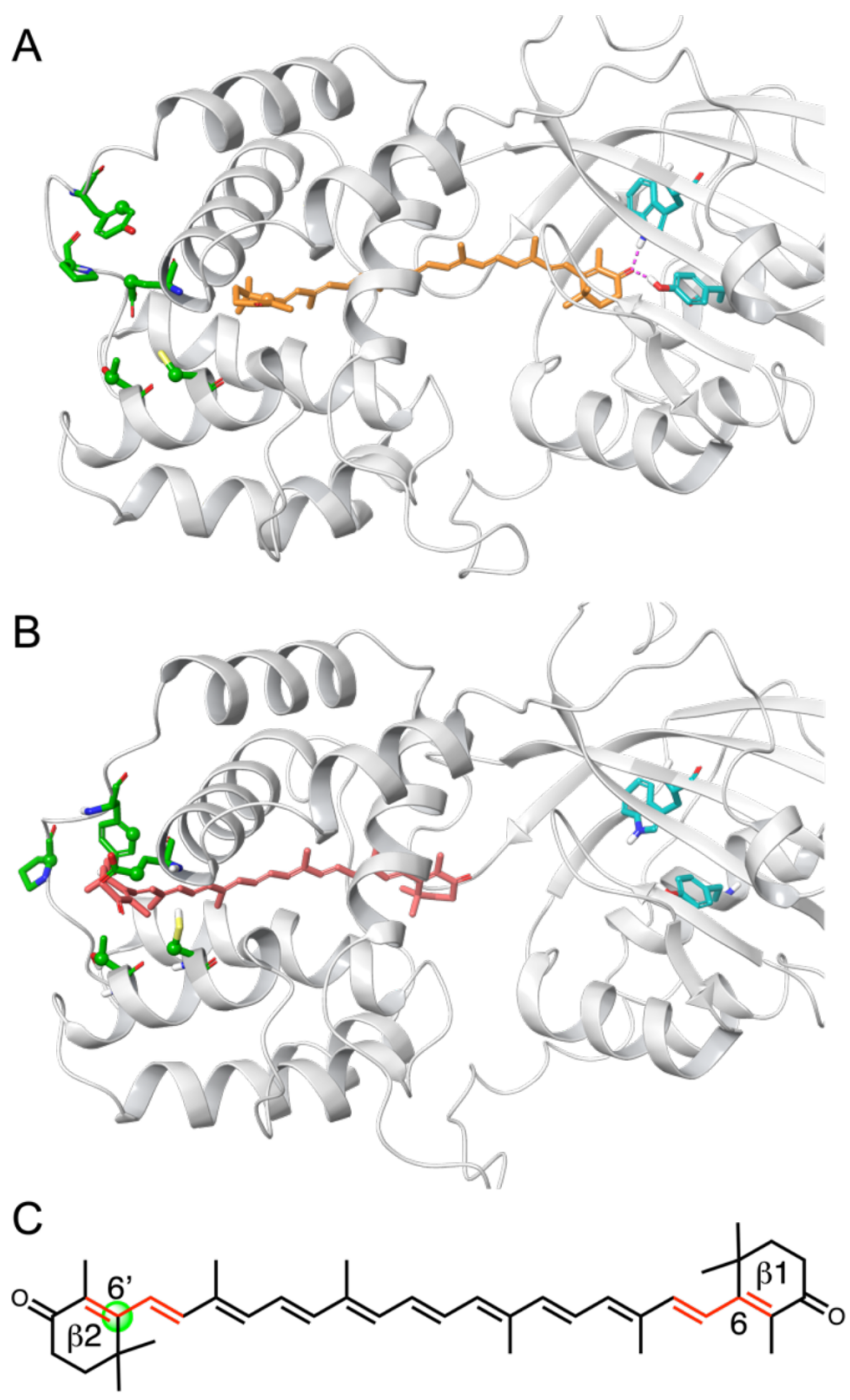

Figure 3. Collective variable (CV) used in WT-metadynamics shown in $\mathrm{OCP}^{\mathrm{O}}$ (A) defined by the distance between the center of mass of the five colored residues (green) in the NTD, and C6 $6^{\prime}$ in CAN's $\beta 2$ ring (C). Resulting $\mathrm{OCP}(\mathrm{P} 3)$ model with $\mathrm{CAN}$ (red color) translocated (B).

thermodynamically accessible conformations that CAN adopts in $\mathrm{RCP}^{16}$ On the other hand, our results for the $\mathrm{OCP}^{\mathrm{O}}$ optimized snapshots from the MD show a predominant $s$-cis configuration for $\beta 2$-dihedral $\left(-45^{\circ} \pm 5^{\circ}\right)$, together with a $\beta 1$ dihedral showing a higher variation but with a more prevalent $s$-trans conformation $\left(112^{\circ} \pm 21^{\circ}\right)$. Previous anisotropy measurements by Konold et al. have also shown that the $\beta 1$ dihedral assumes an $s$-trans conformation in $\mathrm{OCP}^{\mathrm{O}}$. Interestingly, our MD simulation indicates that this nominally $s$-trans $\beta 1$-dihedral is restricted by the protein environment at the CTD of $\mathrm{OCP}^{\mathrm{O}}$ to a maximum angle of $145^{\circ}$. This prevents the enhancement of conjugation in the chromophore, reflected in the orange coloration of the inactive state. Table S1 presents additional analysis of dihedrals and a comparison with simulations in solution.

Metadynamics to Drive Carotenoid Translocation. As pointed out in the Introduction, the photoactivation mechanism in OCP involves a $12 \AA$ carotenoid translocation into the NTD, followed by domain separation. The results from our MD simulation, as well as inferences reported by other authors, ${ }^{16}$ clearly indicate that $\mathrm{OCP}^{\mathrm{O}}$ is a thermodynamically stable complex requiring enhanced sampling methodologies to force the system away from this local energy minimum. Hence, we designed a metadynamics experiment with the aim to drive CAN translocation in OCP bypassing the creation of the photoproduct P1.

In metadynamics, a history-dependent bias potential is built during the simulation as a sum of repulsive Gaussians in the defined collective variable/s (CV) space. Moreover, WTmetadynamics adds a bias factor $(\gamma)$ that limits the exploration to thermodynamically relevant configurations. ${ }^{31}$ The careful selection of the $\mathrm{CV}$ is one of the most important considerations in metadynamics. It can include diverse geometrical descriptors, such as distances, angles, or dihedrals. In the case of OCP, the availability of two reference models representing initial $\left(\mathrm{OCP}^{\mathrm{O}}\right)$ and final $(\mathrm{RCP})$ states of the system facilitates the definition of the $\mathrm{CV}$. In order to force the displacement of the carotenoid toward the NTD using $\mathrm{OCP}^{\mathrm{O}}$ as the starting point, we needed to find a $\mathrm{CV}$ able to drive carotenoid translocation through the tunnel connecting the two domains. The goal was to apply a subtle force to steer CAN into the NTD. A close inspection of the carotenoid in RCP led us to select the CV of our system as the distance between the center of mass of 5 residues on the $\beta 2$-side of the NTD (shown in green in Figure $3 \mathrm{~A}$ ), and the atom $\mathrm{C6}^{\prime}$ of CAN's $\beta 2$-ring (Figure 3C).

It is important to state that we made no attempt to obtain a converged free-energy profile for such a complex reaction path. We used metadynamics only to create reasonable structural models that mimic the intermediate and final states of the translocation process. Indeed, out of 20 repetitions of the experiment, varying the initial seed randomly, the carotenoid did not move from its initial pocket in seven cases. The 13 remaining experiments, for which the translocation of CAN toward the NTD was observed, shared common structural features representing different states along the translocation pathway. Only in two of these experiments did the carotenoid reach a full $12 \AA$ displacement without showing high distortions of the polyene chain. The infrequent occurrence of reasonable paths for translocation suggests that the protein environment effectively limits the movement of the carotenoid. The analysis of characteristic structural features at different points of the simulation is reported here for one of these two paths. The other path, which leads to the same general conclusions, is presented in the Supporting Information (Figure S7). Both of these paths show a similar energy barrier $(\sim 10 \mathrm{kcal} / \mathrm{mol})$ for the overall process of translocation (Figure S8).

Snapshots along the selected path were extracted from the resulting metadynamics trajectory every $1 \mathrm{~ns}$. Each snapshot was optimized at the QM/MM level, using the same procedure described before for the MD snapshots, followed by the calculation of $\lambda_{\max }$. The upper graph in Figure 4 shows that, during the first $48 \mathrm{~ns}$, the excitation energies fluctuate around values similar to those calculated for $\mathrm{OCP}^{\mathrm{O}}$. The solid black line represents the moving average of 5 adjacent snapshots displayed to guide the eye. It is important to mention that metadynamics time does not correspond to the real kinetics of the process. We only use it here to reference different states of the system throughout the path. A significant rise of $\lambda_{\max }$ is observed at $49 \mathrm{~ns}$, with average values reaching the interval of variation obtained for RCP. At this stage, the carotenoid has been displaced halfway between the starting position and the 

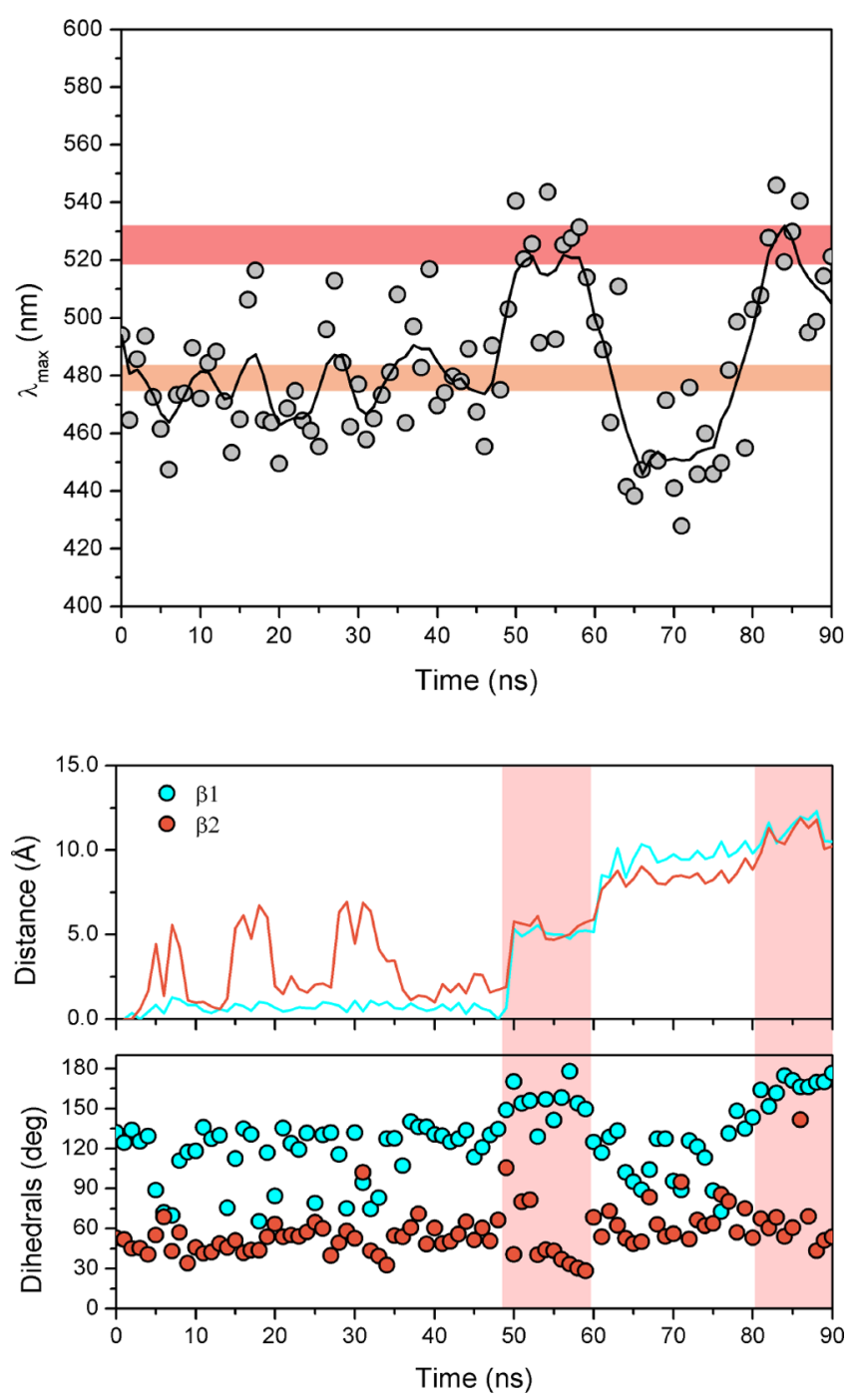

Figure 4. WT-metadynamics results. Calculated $\lambda_{\max }$ values for each optimized snapshot are shown with circles in the upper graph. The solid black line represents a smoothing every 5 points using the adjacent-averaging method. Orange and red stripes define the reference ranges calculated from classical MDs for $\mathrm{OCP}^{\mathrm{O}}$ and $\mathrm{RCP}$, respectively. Lower graphs show the evolution of the distance traveled by C6 ( $\beta 1$-ring) and C6' ( $\beta 2$-ring) of each optimized snapshot with respect to their position at time $0 \mathrm{~ns}$, as well as the absolute dihedral angles connecting the two rings with the isoprenoid chain. Vertical pink stripes highlight the red-shifted intermediate states proposed as photoproducts.

expected final configuration, 5-6 $\mathrm{A}$ from the first snapshot considering $\mathrm{C} 6$ and $\mathrm{C}^{\prime}$ as the reference atoms (Figure 4, lower panel). Between 60 and $80 \mathrm{~ns}$, a marked blue shift of the absorbance maximum is observed. Finally, after $80 \mathrm{~ns}$, the system reaches the full $12 \AA$ translocation, with $\lambda_{\max }$ values reflecting a red-shifted $O C P^{\mathrm{R}}$-like state.

Spectroscopic features of the carotenoid are essentially affected by the conjugation of the system. BLA is a good indicator of the conjugation, but in the case of $\beta$-carotene-like molecules, torsions of terminal rings further influence conjugation. ${ }^{14}$ In fact, rotation of the terminal rings of CAN around the single bonds $\mathrm{C} 6-\mathrm{C} 7$ (in $\beta 1$ ) and $\mathrm{C}^{\prime}-\mathrm{C} 7^{\prime}$ (in $\beta 2$ ) have been previously described as one of the main degrees of freedom modulating the excitation energy. ${ }^{16}$ Results of our metadynamics demonstrate that while the $\beta 2$-dihedral fluctuates mainly within the absolute values $45-60^{\circ}$, RCPlike $\lambda_{\max }$ values are only observed when the $\beta 1$-dihedral values are over $\sim 150^{\circ}$ (s-trans). When either of these two dihedral angles is $\sim 90^{\circ}$, the conjugation of the system decreases, showing blue-shifted states. As mentioned in the previous section, it is clear that the protein environment in different locations inside OCP allows different conformations of the rings, directly affecting the electronic structure of the chromophore.

The resulting WT-metadynamics trajectory can be divided in four main stages representing different locations of CAN in OCP. Two of these states present higher average $\lambda_{\max }$ values, typical of the OCP photoproducts (highlighted by the pink vertical stripes in the lower graphs of Figure 4). Representative snapshots of each state are shown in Figure 5, where the nearest residues (up to $4 \AA$ ) defining the ligand pocket are highlighted. The protein surface helps visualize the shape of the cavities as well as solvent accessibility areas in the proximities of CAN. Ligand SASAs at each stage of the metadynamics trajectory are included in the SI (Table S3). The position represented by the snapshot at 52 ns shows that when the carotenoid is located halfway between initial and final states of the translocation, with the key hydrogen bonds already broken, the protein environment can easily accommodate the chromophore allowing a more planar configuration of the system. We propose this state to be a representative of the $\mathrm{OCP}(\mathrm{P} 2)$ photoproduct. On the contrary, at a distance of $\sim 10 \AA$ from the starting point $(60-80 \mathrm{~ns})$, most of the snapshots show a carotenoid highly distorted from planarity, as exemplified by the snapshot at $70 \mathrm{~ns}$. At this point, isomerization ( $s$-cis) of different bonds along the central torsions of the polyene chain is observed (Table S4) due to constraints by the local protein environment, causing a marked decrease in the conjugation of the system. We propose that such states represent the bottleneck of the translocation process. Finally, after $80 \mathrm{~ns}$, the system reaches a location where, again, a more planar configuration of the carotenoid is allowed. At this point, the location of CAN within the cavity is very similar to that observed in the RCP crystal (Figure 6). The outstanding difference found between both models is related to the configuration of both end-ring torsions. Interestingly, as has been demonstrated by Mennucci et al., there are several thermodynamically stable conformations found for CAN in RCP besides the one observed in the crystal structure. ${ }^{16}$ In summary, we propose that states like these are models of the OCP(P3) photoproduct.

Red-Shifted Form of OCPO . The heterogeneity observed experimentally in the UV-vis absorbance spectra of $\mathrm{OCP}^{\mathrm{O}}$ bound to different carotenoids, showing two bands of similar strength (at 477 and $500 \mathrm{~nm}$ for OCP-CAN), has been initially attributed to either the presence of $\mathrm{OCP}^{\mathrm{R}}$ in the sample, ${ }^{32}$ or to the existence of an alternative form of $\mathrm{OCP}^{\mathrm{O}} \cdot{ }^{33}$ More recent experimental evidence supports the second hypothesis establishing the existence of a "red-shifted" form of $\mathrm{OCP}^{\mathrm{O}} \cdot{ }^{14,15}$ According to these authors, this form should be characterized by a looser binding pocket, with a higher degree of conjugation of the carotenoid's isoprenoid chain. Despite these inferences, a detailed structural characterization of this "red-shifted" state has remained elusive. The results of our WT-metadynamics trajectory show a subpopulation of redshifted snapshots distributed along the time range between 0 and 48 ns which are still bound to Tyr201 and Trp288 via hydrogen bonds. This observation leads us to propose this 

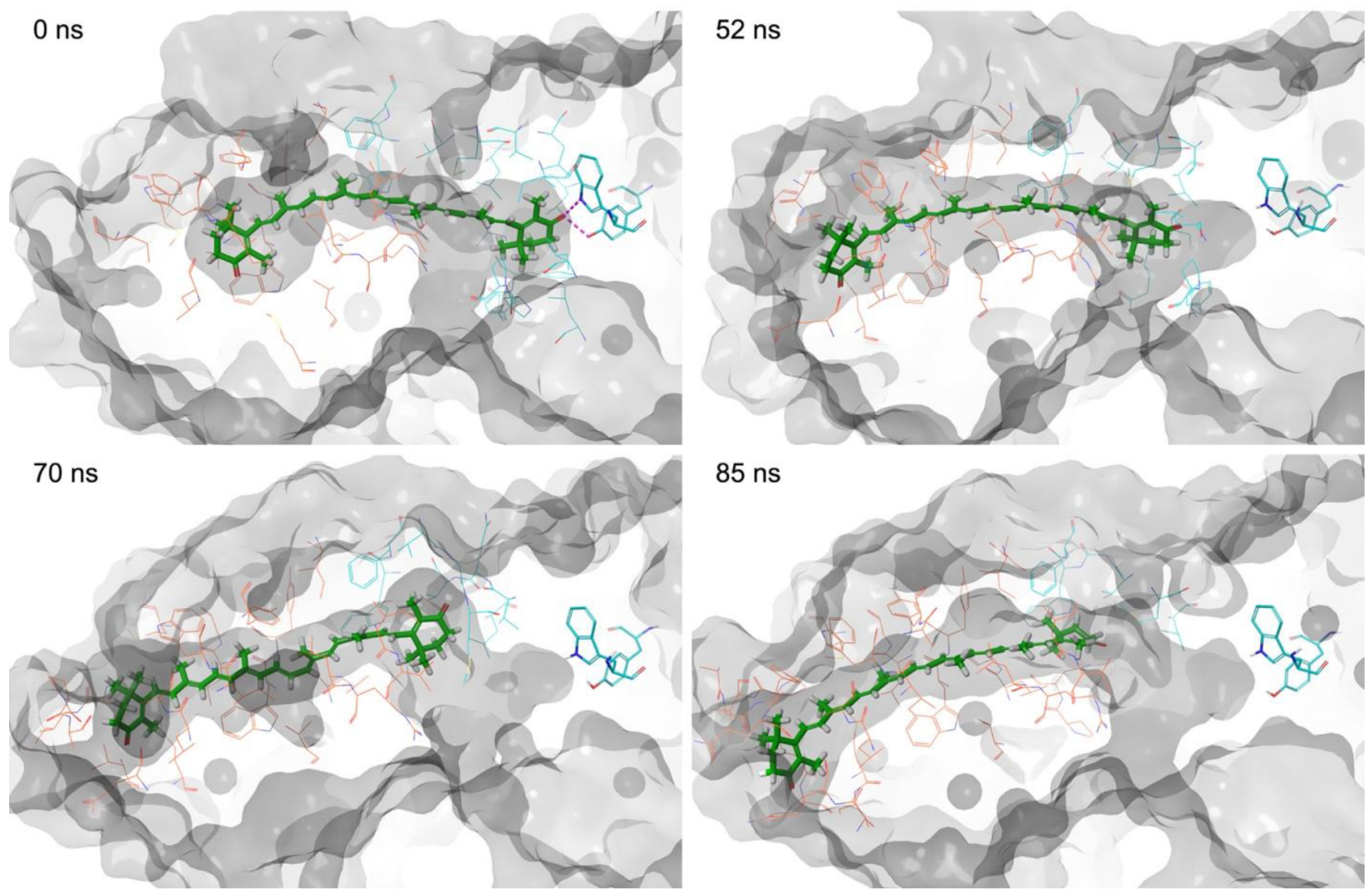

Figure 5. Representative snapshots at different stages of the WT-metadynamics trajectory, starting with $\mathrm{OCP}^{\mathrm{O}}(0 \mathrm{~ns})$. NTD residues are colored in orange, and CTD residues, in cyan. Tyr201 and Trp288 are highlighted with sticks, and hydrogen-bonding interactions are indicated with magenta dashed lines. The semitransparent surfaces are the protein surfaces defined by the protein residues (excluding CAN). The darker regions represent cavities that are accessible to CAN and solvent.

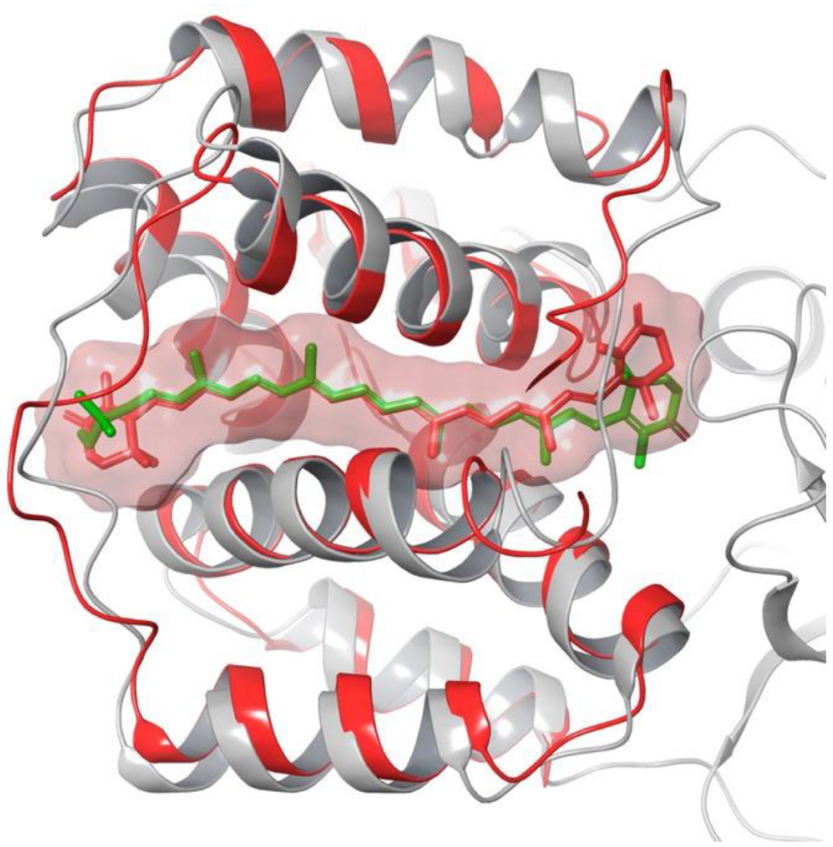

Figure 6. Superposition of RCP crystal structure (red ribbons, CAN in red) with the resulting $\mathrm{OCP}(\mathrm{P} 3)$ model from the WTmetadynamics experiment (gray ribbons, $\mathrm{CAN}$ in green). The red surface corresponds to the cavity defined by the ligand in RCP.

subpopulation of structures as the hypothetical "red-shifted" form of $\mathrm{OCP}^{\mathrm{O}}$. The snapshot at 17 ns (Figure 7) represents an example of this alternative form where the protein environment is reorganized enabling a more planar configuration of the conjugated chain when compared to the main form of $\mathrm{OCP}^{\mathrm{O}}(0 \mathrm{~ns})$. As can be observed, the key hydrogen-bonding interactions with Tyr201 and Trp288 are kept unaltered in such a state. However, the occurrence of this "red-shifted" form involves an excursion into the NTD side allowing for the $\beta 2$-end to stick out of the protein. This is consistent with the observation of the N-terminal domain being much more flexible than the C-terminal domain. ${ }^{30}$

\section{CONCLUSIONS}

Using metadynamics, we have obtained insight into the process of carotenoid translocation in the photoactivation mechanism of OCP. We have proposed approximate molecular models for the two intermediate photoproducts (P2 and P3). To the best of our knowledge, this is the first study that simulates chromophore displacement and its relationship to absorption spectroscopy. The close correlation of the calculated values of the proposed intermediates with the UV-vis features reported from experimental studies gives a reasonable validation to our models. The finding of a single geometric variable capable of producing a plausible reaction path offers an important contribution for future characterization of OCP with various carotenoid chromophores. We have developed a computational methodology combining MD and QM/MM calculations which allowed us to reproduce the experimental $\lambda_{\max }$ values of the reference models ( $\mathrm{OCP}^{\mathrm{O}}$ and $\left.\mathrm{RCP}\right)$. In addition, we were able to propose a model of a red-shifted form of $\mathrm{OCP}^{\mathrm{O}}$, 


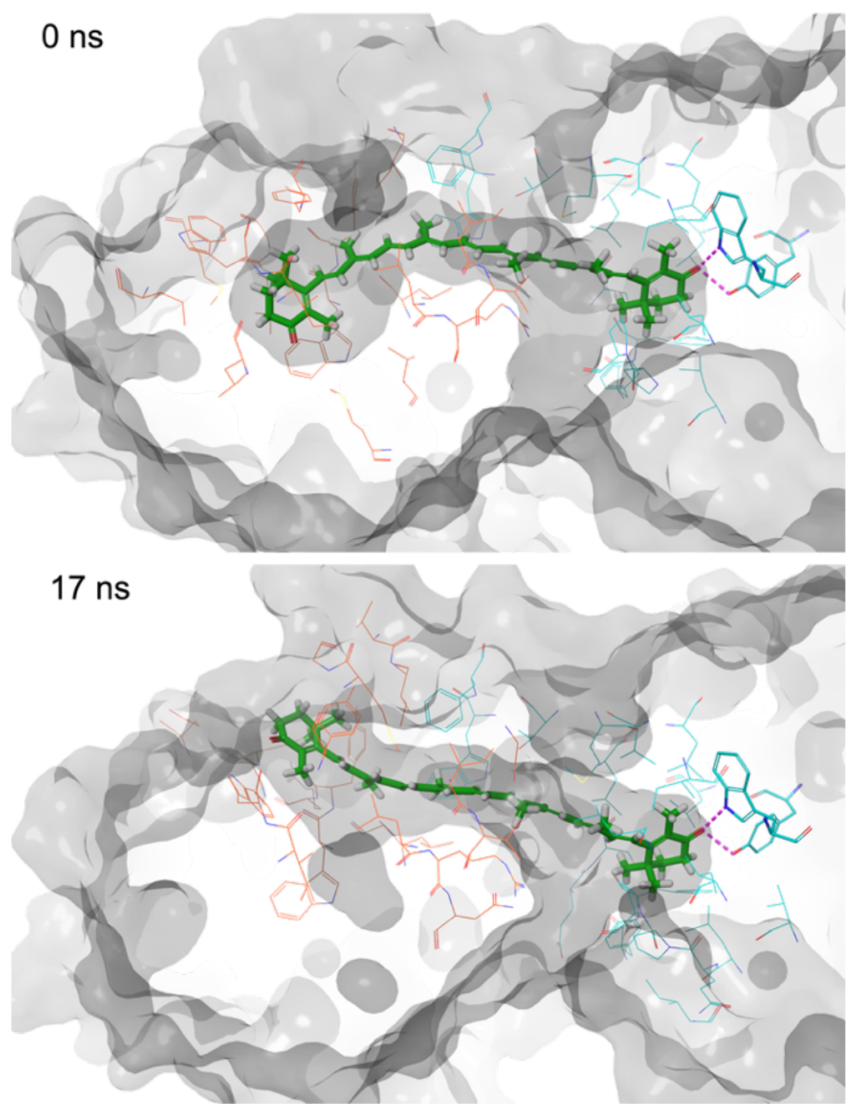

Figure 7. Representative snapshot of the hypothetical "red-shifted" form of $\mathrm{OCP}^{\mathrm{O}}$ (at $17 \mathrm{~ns}$ ). NTD residues are colored in orange, and CTD residues, in cyan. Tyr201 and Trp288 are highlighted with thicker sticks, and hydrogen-bonding interactions are indicated with magenta dashed lines. The conformation of CAN in the standard blue-shifted state (at $0 \mathrm{~ns}$ ) is shown for comparison.

previously inferred by spectroscopy experiments. Although the primary event of OCP photoactivation (the formation of the P1 photoproduct) is still elusive, our work presents new insight into how the protein environment exerts distortions on the carotenoid and indirectly modulates the electronic properties. The OCP(P3) model shows a high resemblance to RCP and presents a useful starting point to explore the following step of the mechanism, protein domain separation. Current work in our laboratory is aiming to model this process.

\section{ASSOCIATED CONTENT}

\section{SI Supporting Information}

The Supporting Information is available free of charge at https://pubs.acs.org/doi/10.1021/acs.jpcb.0c08756.

Additional data for the $\mathrm{MD}$ procedure (relaxation protocol) and $\mathrm{MD}$ analysis (RMSD, protein-ligand interactions, ligand SASA, and end-ring torsions); BLA calculation method together with its correlation with $\lambda_{\max }$ values; and additional data of the metadynamics analysis, as well as results of the metadynamics repetition, together with the free-energy profile of both experiments (PDF)

\section{AUTHOR INFORMATION}

\section{Corresponding Author}

José A. Gascón - Department of Chemistry, University of Connecticut, Storrs, Connecticut 06269-3060, United States; ○ orcid.org/0000-0002-4176-9030; Email: jose.gascon@ uconn.edu

\section{Authors}

Natalia B. Pigni - Department of Chemistry, University of Connecticut, Storrs, Connecticut 06269-3060, United States; Instituto de Ciencia y Tecnología de Alimentos Córdoba (ICYTAC-CONICET), Ciudad Universitaria, Córdoba X5000HUA, Argentina

Kevin L. Clark - Department of Chemistry, University of Connecticut, Storrs, Connecticut 06269-3060, United States

Warren F. Beck - Department of Chemistry, Michigan State University, East Lansing, Michigan 48824-1322, United States; (1) orcid.org/0000-0002-7491-0264

Complete contact information is available at:

https://pubs.acs.org/10.1021/acs.jpcb.0c08756

\section{Notes}

The authors declare no competing financial interest.

\section{ACKNOWLEDGMENTS}

This work was supported by NSF Grants CHE-1904700 to J.A.G. and CHE-1904655 to W.F.B. Additional support for the laboratory of W.F.B. was provided by award DE-SC0010847 from the Photosynthetic Systems program of the Chemical Sciences, Geosciences and Biosciences Division, Office of Basic Energy Sciences, Office of Science, of the U.S. Department of Energy.

\section{REFERENCES}

(1) Kirilovsky, D.; Kaňa, R.; Prášil, O. Mechanisms Modulating Energy Arriving at Reaction Centers in Cyanobacteria. In NonPhotochemical Quenching and Energy Dissipation in Plants, Algae and Cyanobacteria; Demmig-Adams, B.; Garab, G.; Adams, W., III; Govindjee, Eds.; Springer Netherlands: Dordrecht, 2014; pp 471501.

(2) Wilson, A.; Punginelli, C.; Gall, A.; Bonetti, C.; Alexandre, M.; Routaboul, J.-M.; Kerfeld, C. A.; van Grondelle, R.; Robert, B.; Kennis, J. T. M.; Kirilovsky, D. A photoactive carotenoid protein acting as light intensity sensor. Proc. Natl. Acad. Sci. U. S. A. 2008, 105 (33), 12075.

(3) Kerfeld, C. A.; Sawaya, M. R.; Brahmandam, V.; Cascio, D.; Ho, K. K.; Trevithick-Sutton, C. C.; Krogmann, D. W.; Yeates, T. O. The Crystal Structure of a Cyanobacterial Water-Soluble Carotenoid Binding Protein. Structure 2003, 11 (1), 55-65.

(4) Harris, D.; Tal, O.; Jallet, D.; Wilson, A.; Kirilovsky, D.; Adir, N. Orange carotenoid protein burrows into the phycobilisome to provide photoprotection. Proc. Natl. Acad. Sci. U. S. A. 2016, 113 (12), E1655.

(5) Kerfeld, C. A.; Melnicki, M. R.; Sutter, M.; Dominguez-Martin, M. A. Structure, function and evolution of the cyanobacterial orange carotenoid protein and its homologs. New Phytol. 2017, 215 (3), 937-951.

(6) Wilson, A.; Punginelli, C.; Couturier, M.; Perreau, F.; Kirilovsky, D. Essential role of two tyrosines and two tryptophans on the photoprotection activity of the Orange Carotenoid Protein. Biochim. Biophys. Acta, Bioenerg. 2011, 1807 (3), 293-301.

(7) Gurchiek, J. K.; Bao, H.; Domínguez-Martín, M. A.; McGovern, S. E.; Marquardt, C. E.; Roscioli, J. D.; Ghosh, S.; Kerfeld, C. A.; Beck, W. F. Fluorescence and Excited-State Conformational Dynamics of the Orange Carotenoid Protein. J. Phys. Chem. B 2018, 122 (6), $1792-1800$ 
(8) Konold, P. E.; van Stokkum, I. H. M.; Muzzopappa, F.; Wilson, A.; Groot, M.-L.; Kirilovsky, D.; Kennis, J. T. M. Photoactivation Mechanism, Timing of Protein Secondary Structure Dynamics and Carotenoid Translocation in the Orange Carotenoid Protein. J. Am. Chem. Soc. 2019, 141 (1), 520-530.

(9) Leverenz, R. L.; Sutter, M.; Wilson, A.; Gupta, S.; Thurotte, A.; Bourcier de Carbon, C.; Petzold, C. J.; Ralston, C.; Perreau, F.; Kirilovsky, D.; Kerfeld, C. A. A 12 A carotenoid translocation in a photoswitch associated with cyanobacterial photoprotection. Science 2015, 348 (6242), 1463.

(10) Gupta, S.; Guttman, M.; Leverenz, R. L.; Zhumadilova, K.; Pawlowski, E. G.; Petzold, C. J.; Lee, K. K.; Ralston, C. Y.; Kerfeld, C. A. Local and global structural drivers for the photoactivation of the orange carotenoid protein. Proc. Natl. Acad. Sci. U. S. A. 2015, 112 (41), E5567.

(11) Gupta, S.; Sutter, M.; Remesh, S. G.; Dominguez-Martin, M. A.; Bao, H.; Feng, X. A.; Chan, L.-J. G.; Petzold, C. J.; Kerfeld, C. A.; Ralston, C. Y. X-ray radiolytic labeling reveals the molecular basis of orange carotenoid protein photoprotection and its interactions with fluorescence recovery protein. J. Biol. Chem. 2019, 294 (22), 88488860 .

(12) Liu, H.; Zhang, H.; King, J. D.; Wolf, N. R.; Prado, M.; Gross, M. L.; Blankenship, R. E. Mass spectrometry footprinting reveals the structural rearrangements of cyanobacterial orange carotenoid protein upon light activation. Biochim. Biophys. Acta, Bioenerg. 2014, 1837 (12), 1955-1963.

(13) Liu, H.; Zhang, H.; Orf, G. S.; Lu, Y.; Jiang, J.; King, J. D.; Wolf, N. R.; Gross, M. L.; Blankenship, R. E. Dramatic Domain Rearrangements of the Cyanobacterial Orange Carotenoid Protein upon Photoactivation. Biochemistry 2016, 55 (7), 1003-1009.

(14) Šlouf, V.; Kuznetsova, V.; Fuciman, M.; de Carbon, C. B.; Wilson, A.; Kirilovsky, D.; Polívka, T. Ultrafast spectroscopy tracks carotenoid configurations in the orange and red carotenoid proteins from cyanobacteria. Photosynth. Res. 2017, 131 (1), 105-117.

(15) Kuznetsova, V.; Dominguez-Martin, M. A.; Bao, H.; Gupta, S.; Sutter, M.; Kloz, M.; Rebarz, M.; Přeček, M.; Chen, Y.; Petzold, C. J.; Ralston, C. Y.; Kerfeld, C. A.; Polívka, T. Comparative ultrafast spectroscopy and structural analysis of OCP1 and OCP2 from Tolypothrix. Biochim. Biophys. Acta, Bioenerg. 2020, 1861 (2), 148120.

(16) Bondanza, M.; Cupellini, L.; Lipparini, F.; Mennucci, B. The Multiple Roles of the Protein in the Photoactivation of Orange Carotenoid Protein. Chem. 2020, 6 (1), 187-203.

(17) Barducci, A.; Bonomi, M.; Parrinello, M. Metadynamics. Wiley Interdiscip. Rev.: Comput. Mol. Sci. 2011, 1 (5), 826-843.

(18) Branduardi, D.; Gervasio, F. L.; Cavalli, A.; Recanatini, M.; Parrinello, M. The Role of the Peripheral Anionic Site and Cation $-\pi$ Interactions in the Ligand Penetration of the Human AChE Gorge. J. Am. Chem. Soc. 2005, 127 (25), 9147-9155.

(19) Jorgensen, W. L.; Chandrasekhar, J.; Madura, J. D.; Impey, R. W.; Klein, M. L. Comparison of simple potential functions for simulating liquid water. J. Chem. Phys. 1983, 79 (2), 926-935.

(20) Roos, K.; Wu, C.; Damm, W.; Reboul, M.; Stevenson, J. M.; Lu, C.; Dahlgren, M. K.; Mondal, S.; Chen, W.; Wang, L.; Abel, R.; Friesner, R. A.; Harder, E. D. OPLS3e: Extending Force Field Coverage for Drug-Like Small Molecules. J. Chem. Theory Comput. 2019, 15 (3), 1863-1874.

(21) Chábera, P.; Fuciman, M.; Hř́bek, P.; Polívka, T. Effect of carotenoid structure on excited-state dynamics of carbonyl carotenoids. Phys. Chem. Chem. Phys. 2009, 11 (39), 8795-8803.

(22) Khan, T.; Dominguez-Martin, M. A.; Śímová, I.; Fuciman, M.; Kerfeld, C. A.; Polívka, T. Excited-State Properties of Canthaxanthin in Cyanobacterial Carotenoid-Binding Proteins HCP2 and HCP3. J. Phys. Chem. B 2020, 124 (24), 4896-4905.

(23) Andreussi, O.; Knecht, S.; Marian, C. M.; Kongsted, J.; Mennucci, B. Carotenoids and Light-Harvesting: From DFT/MRCI to the Tamm-Dancoff Approximation. J. Chem. Theory Comput. 2015, 11 (2), 655-666.

(24) Spezia, R.; Knecht, S.; Mennucci, B. Excited state characterization of carbonyl containing carotenoids: a comparison between single and multireference descriptions. Phys. Chem. Chem. Phys. 2017, 19 (26), 17156-17166.

(25) Gurchiek, J. K.; Rose, J. B.; Guberman-Pfeffer, M. J.; Tilluck, R. W.; Ghosh, S.; Gascón, J. A.; Beck, W. F. Fluorescence anisotropy detection of barrier crossing and ultrafast conformational dynamics in the S2 state of $\beta$-carotene. J. Phys. Chem. B 2020, 124, 9029.

(26) Guberman-Pfeffer, M. J.; Greco, J. A.; Birge, R. R.; Frank, H. A.; Gascón, J. A. Light Harvesting by Equally Contributing Mechanisms in a Photosynthetic Antenna Protein. J. Phys. Chem. Lett. 2018, 9 (3), $563-568$

(27) Prandi, I. G.; Viani, L.; Andreussi, O.; Mennucci, B. Combining classical molecular dynamics and quantum mechanical methods for the description of electronic excitations: The case of carotenoids. $J$. Comput. Chem. 2016, 37 (11), 981-991.

(28) Bondanza, M.; Nottoli, M.; Cupellini, L.; Lipparini, F.; Mennucci, B. Polarizable embedding QM/MM: the future gold standard for complex (bio)systems? Phys. Chem. Chem. Phys. 2020, 22 (26), 14433-14448.

(29) Wei, T.; Balevičius, V.; Polívka, T.; Ruban, A. V.; Duffy, C. D. P. How carotenoid distortions may determine optical properties: lessons from the Orange Carotenoid Protein. Phys. Chem. Chem. Phys. 2019, 21 (41), 23187-23197.

(30) Bandara, S.; Ren, Z.; Lu, L.; Zeng, X.; Shin, H.; Zhao, K.-H.; Yang, X. Photoactivation mechanism of a carotenoid-based photoreceptor. Proc. Natl. Acad. Sci. U. S. A. 2017, 114 (24), 6286.

(31) Bussi, G.; Branduardi, D. Free-Energy Calculations with Metadynamics: Theory and Practice. Reviews in Computational Chemistry 2015, 28, 1-49.

(32) Polívka, T.; Chábera, P.; Kerfeld, C. A. Carotenoid-protein interaction alters the $\mathrm{S} 1$ energy of hydroxyechinenone in the Orange Carotenoid Protein. Biochim. Biophys. Acta, Bioenerg. 2013, 1827 (3), 248-254.

(33) Kish, E.; Pinto, M. M. M.; Kirilovsky, D.; Spezia, R.; Robert, B. Echinenone vibrational properties: From solvents to the orange carotenoid protein. Biochim. Biophys. Acta, Bioenerg. 2015, 1847 (10), 1044-1054 